\title{
AN INFLUENCE OF 7.5 T SUPERCONDUCTING WIGGLER ON BEAM PARAMETERS OF SIBERIA-2 STORAGE RING
}

\author{
V. Korchuganov, A. Valentinov, Kurchatov Institute, Moscow, Russia \\ N. Mezentsev, BINP, Novosibirsk, Russia
}

\begin{abstract}
At present the dedicated synchrotron radiation source Siberia-2 in Kurchatov Institute operates with electron energy $2.5 \mathrm{GeV}$ and current up to $200 \mathrm{~mA}$. In order to expand spectral range of SR and to increase brightness an installation of $7.5 \mathrm{~T}$ 19-pole superconducting wiggler is planned at the end of 2006. Now the wiggler is under fabrication in BINP, Novosibirsk. Such high level of a magnetic field in the wiggler will have a great influence on electron beam parameters of Siberia-2. Changes of these parameters (betatron tunes, horizontal emittance of the electron beam, momentum compaction, energy spread etc.) are discussed in the report. Different methods of compensation (global and local) of betatron functions distortion are presented. Much attention is paid to dynamic aperture calculations using analytical approximation of magnetic field behavior in transverse horizontal.
\end{abstract}

\section{INTRODUCTION}

Dedicated synchrotron radiation (SR) source Siberia-2 [1] operates regularly for users since 1999. Working energy of Siberia-2 is $2.5 \mathrm{GeV}$, electron beam current achieved $200 \mathrm{~mA}$. SR users get radiation from bending magnets with $1.7 \mathrm{~T}$ field level. In order to extend spectral range and to raise brightness of synchrotron radiation a superconducting wiggler with maximum field $7.5 \mathrm{~T}$ will be installed on the ring at the end of 2006. It will supply 3 hard X-rays beam lines. The minimum critical wavelength of wiggler radiation is equal to $0.4 \AA$ while this value for bending magnets equals $1.75 \AA$. 19 poles wiggler will provide powerful spectral flux and brightness of SR. Figure 1 demonstrates SR spectral flux (normalized for 1 Ampere electron current) from wiggler and bending magnet. General features of wiggler design and calculations of wiggler influence on beam parameters are presented below.

\section{WIGGLER DESIGN}

Magnet system of the wiggler contains 21 poles - 19 ones with maximum field and 2 with half-value of this field. Each pole consists of two superconducting coils and ARMKO yoke. Coils are situated symmetrically relative to vacuum chamber in vertical plane. Poles on every side of the vacuum chamber are fixed on a long $1800 \mathrm{~mm}$ steel plate. The plate closes magnet fields and simultaneously serves as a rigid support for poles. The vacuum chamber is a part of helium vessel and defines the distance between upper and lower poles. Nb-Ti wire with $\varnothing 0.92$ $\mathrm{mm}$ (in isolation) was used to form the coils. Each coil is divided in two electrically independent sections; second section is winded over first one. All inner sections are connected in series, as well as outer sections. To optimize field/current relation two power supplies are used. Inner sections are fed by first power supply while outer sections are fed by sum of currents of both power supplies. Side poles of the wiggler have only one section in their coils and also are fed by sum of the currents. This scheme permits to control field integrals very easy. General parameters of the wigglers are presented in Table 1.

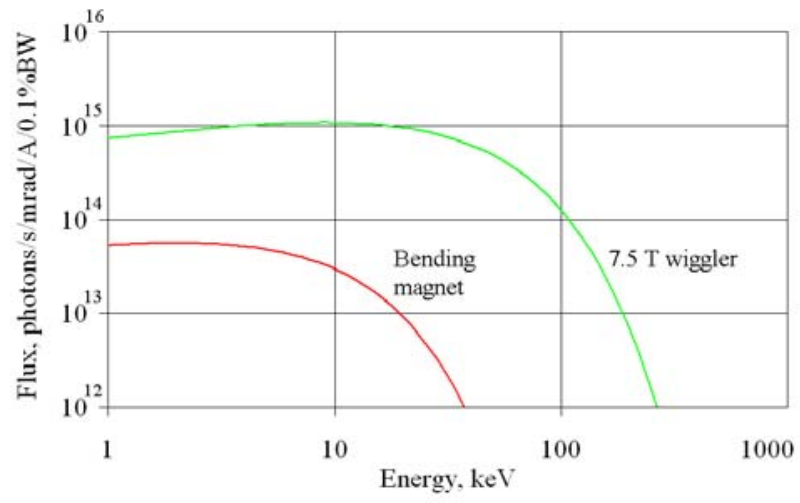

Figure 1: Spectral flux of synchrotron radiation from bending magnet of Siberia-2 and 7.5 $\mathrm{T}$ superconducting wiggler.Layout of papers.

Table 1: General parameters of the wiggler

\begin{tabular}{|l|l|}
\hline Number of the poles & $19+2$ \\
\hline Maximum magnetic field & $7.5 \mathrm{~T}$ \\
\hline Wiggler period & $164 \mathrm{~mm}$ \\
\hline Gap between upper and lower poles & $20.2 \mathrm{~mm}$ \\
\hline Vacuum chamber gap & $13 \mathrm{~mm}$ \\
\hline Pole height $\times$ width $\times$ length & $75.8 \times 236 \times 82 \mathrm{~mm}$ \\
\hline Number of turns in one section & 774 \\
\hline Currents of power supplies at $7.5 \mathrm{~T}$ & $180-200 \mathrm{~A}$ \\
\hline Maximum stored energy in wiggler & $500 \mathrm{~kJ}$ \\
\hline
\end{tabular}

Magnet system of the wiggler is situated inside a cryostat. Outer body of cryostat is fabricated from $6 \mathrm{~mm}$ thick stainless steel and has cylinder-like form. Distance between opposite vacuum chamber flanges is $2400 \mathrm{~mm}$. To cool the wiggler and to keep temperature conditions during its operation four two-stage coolers (made in "Leybold") are used. Two coolers Coolpower $10 \mathrm{MD}$ are connected to copper screens with temperatures $60^{\circ} \mathrm{K}$ and $20^{\circ} \mathrm{K}$ and copper liner inside vacuum chamber. Two coolers Coolpower 4.2GM One Watt System are 
responsible for cooling of $60^{\circ} \mathrm{K}$ and $20^{\circ} \mathrm{K}$ main and auxiliary leads, cooper screens and $4^{0} \mathrm{~K}$ helium vessel. All coolers are driven by compressor units COOLPACK 6200 MD. Helium vessel is hanged inside the cryostat on kevlar strips in order to minimize heat flows. For the same reason low pressure of $10^{-7}$ Torr is supported inside the cryostat. Input conductors consist of the brass rods and high temperature superconducting ceramics (produced by “American Superconductors").

Special equipment was designed in BINP for a wiggler control system using VME standard. Wiggler is controlled by a separate PC, but the connection with control system of the ring is also available. Sensors inside the cryostat measure the temperature, liquid He level, pressure of He gas and vacuum level. Interlock systems are available for the case of quenches, abnormal temperature growth, low level of liquid He. Magnetic field is controlled by simultaneous change of two power supplies' currents. Preliminary magnetic measurements should be done in order to provide zero value of first field integral along the wiggler for all field values. Software consists of two parts: first computer program works in VME crate under VxWorks 5.3 operational system, second one operates in PC under MS Windows. Software in VME crate can act autonomously in the case of necessity. Program in PC allows controlling wiggler field, represents current status of cryostat and power supplies. Similar control systems now are in operation in several acceleration centers (BESSY, CLS, ELETTRA) [2-4].

\section{WIGGLER FABRICATION}

Wiggler fabrication now is going on in BINP. Magnet system of the wiggler is ready, manufacturing of the cryostat is in progress. In order to check wiggler construction 5-pole wiggler prototype was made in 2005. Field level 7.6 $\mathrm{T}$ was achieved after training of the prototype. Spatial distribution of the magnetic field is close to predicted one. Manufacturing and magnetic measurements of the wiggler will be finished in September 2006. Then wiggler will be transported to Kurchatov Institute and magnetic measurement will be done again. The wiggler will be installed on the ring at the end of 2006.

\section{WIGGLER INFLUENCE ON BEAM PARAMETERS IN SIBERIA-2}

Strong wiggler field has a great influence on beam dynamics in the storage ring. To minimize an effect of the wiggler presence, it will be situated in $3.0 \mathrm{~m}$ long straight section with zero dispersion and optimal values of betatron functions $\left(\beta_{\mathrm{x}}=4.1 \mathrm{~m}\right.$ and $\beta_{\mathrm{z}}=0.59 \mathrm{~m}$ in the center of the straight section). Except wiggler the straight section also includes bellows, SR absorbers and special vacuum chambers providing smooth transition from elliptical profile of the chamber inside the wiggler to more sophisticated profile in neighboring quadrupole lenses. A position of the wiggler in zero dispersion region allows to increase radiation damping of betatron oscillations and therefore to decrease horizontal emittance of the ring. Low value of $\beta_{\mathrm{z}}$-function minimizes shift of vertical betatron tune. Influence of the wiggler on general beam parameters of Siberia- 2 is presented in Table 2.

Table 2: Wiggler influence on general beam parameters in Siberia-2.

\begin{tabular}{|l|l|l|}
\hline Parameters & $\begin{array}{l}\text { Without } \\
\text { wiggler }\end{array}$ & $\begin{array}{l}\text { With wiggler } \\
7.5 ~ \mathbf{~ T}\end{array}$ \\
\hline Energy, GeV emittance, & 2.5 & 2.5 \\
\hline $\begin{array}{l}\text { Horizontal } \\
\text { nm·rad }\end{array}$ & 98 & 64.7 \\
\hline Betatron tune shifts, $\Delta \mathrm{Q}_{\mathrm{x}, \mathrm{z}}$ & - & $0,0.05$ \\
\hline Radiation loss/turn, keV & 685 & 1041 \\
\hline Momentum compaction & .01036 & .01036 \\
\hline Energy dispertion, $\sigma_{\mathrm{E}} / \mathrm{E}$ & .000953 & .00133 \\
\hline $\begin{array}{l}\text { Damping times, ms } \tau_{\mathrm{x}}, \tau_{\mathrm{z}}, \\
\tau_{\mathrm{s}}\end{array}$ & $\begin{array}{l}3.15,3.02, \\
1.48\end{array}$ & $\begin{array}{l}2.05,1.99, \\
0.98\end{array}$ \\
\hline $\begin{array}{l}\text { RF-voltage amplitude, } \\
\text { MV }\end{array}$ & $\begin{array}{l}1.2 \text { (current } \\
\text { value) }\end{array}$ & $\begin{array}{l}1.61 \text { (for the } \\
\text { same energy } \\
\text { acceptance) }\end{array}$ \\
\hline
\end{tabular}

The wiggler can distort closed orbit of electrons in the ring. Dipole orbit correctors in neighboring quadrupole lenses can compensate orbit deflection of about $0.5 \mathrm{mrad}$ in vertical plane and $1.8 \mathrm{mrad}$ in horizontal plane.

The wiggler creates strong additional focusing and distorts betatron functions of entire ring. The focusing force depends on behavior of vertical magnetic field in horizontal direction. In order to evaluate an wiggler influence a computer modeling was done. Wiggler field components depend on transversal coordinates $\mathrm{x}, \mathrm{z}$ and longitudinal s as follows:

$$
\begin{aligned}
& B_{x}=-\frac{k_{x}}{k_{z}} \cdot B_{0} \cdot \sin \left(k_{x} x\right) \sinh \left(k_{z} z\right) \sin \left(k_{s} s\right) \\
& B_{s}=\frac{k_{s}}{k_{z}} \cdot B_{0} \cdot \cos \left(k_{x} x\right) \sinh \left(k_{z} z\right) \cos \left(k_{s} s\right) \\
& B_{z}=B_{0} \cdot \cos \left(k_{x} x\right) \cosh \left(k_{z} z\right) \sin \left(k_{s} s\right)
\end{aligned}
$$

Here $\mathrm{k}_{\mathrm{s}}=\frac{2 \pi}{\lambda_{\mathrm{w}}}, \mathrm{k}_{\mathrm{x}}=\frac{2 \pi}{\xi}, \quad \xi$ - parameter of field decreasing in horizontal direction, $\mathrm{k}_{\mathrm{z}}^{2}=\mathrm{k}_{\mathrm{x}}^{2}+\mathrm{k}_{\mathrm{s}}^{2}, \mathrm{~B}_{0}$ is field amplitude, $\lambda_{w}$ - wiggler field period in longitudinal direction. Parameter $\xi$ may be calculated from magnetic measurements:

$$
\xi=2 \pi \sqrt{\frac{\mathrm{B}_{0}}{\frac{\partial^{2} \mathrm{~B}_{\mathrm{z}}}{\partial \mathrm{x}^{2}}}} \text { (at the center of the pole) }
$$

Each wiggler pole was divided in thin slices during modeling. New electron trajectory was found and betatron functions were calculated on it. Similar method was used 
for dynamic aperture calculations by tracking. Beam parameters, betatron function and dynamic aperture were calculated as functions of wiggler maximum field and parameter $\xi$. It was found that $\beta$-functions beatings from cell to cell exceed $10 \%$ then $\xi \leq 80 \mathrm{~cm}$. The beatings grow sharply with $\xi$ decreasing.

Betatron tune shifts are very large so they must be compensated by magnet system of the storage ring. There are two ways of the compensation: global (by quadrupole lenses in all cells of the ring) and local (by gradient correction coils in neighboring lenses). Local methods keep dynamic aperture almost constant down to low values of $\xi$ (see Fig.2).
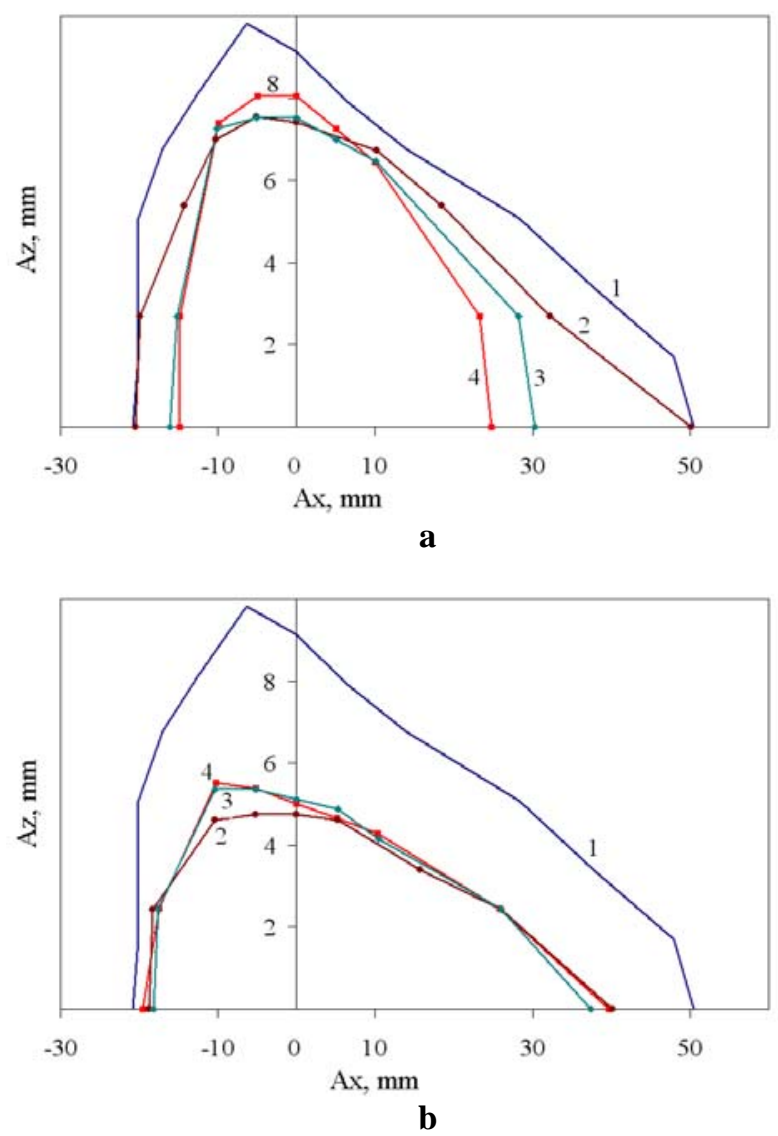

Figure 2: Dynamic aperture of Siberia-2 after compensation of betatron tune shifts. a - global compensation, b - local compensation, 1 - aperture without wiggler, 2 - aperture with parameter $\xi=\infty$ (flat field), 3 - for $\xi=120 \mathrm{~cm}, 4$ - for $\xi=80 \mathrm{~cm}$. Initial point for calculation of the dynamic aperture is situated on the opposite side of the ring relative to the wiggler.
Global methods lead to decreasing of the dynamic aperture for $\xi$ less then $100 \mathrm{~cm}$. Local methods also excite $15-20 \% \beta_{z}$ beatings around the ring.

Unfortunately gradient correction coils are not strong enough to compensate betatron tune shifts at working energy $2.5 \mathrm{GeV}$. But local methods may be useful at injection energy $0.45 \mathrm{GeV}$. Wiggler field will not be switched off during injection process because of strong residual magnetic fields that cannot be controlled properly. As one can see wiggler influence at injection energy corresponds to its influence at $2.5 \mathrm{GeV}$ when the maximum magnetic field is equal to $1.3 \mathrm{~T}$. It leads to low values of power supply currents. A form of the field will be far from sinusoidal one in this case. It is particularly important to have large horizontal dynamic aperture during injection because it takes place in horizontal plane. Local methods may be successfully used in this case.

\section{CONCLUSIONS}

An installation of superconducting wiggler on Siberia-2 storage ring will lead to prominent growth of possibilities for SR users. Magnet system of Siberia-2 can provide compensation of wiggler influence on beam dynamic successfully. An attention should be paid to reliability of RF-system because of higher RF-voltage during wiggler operation. Proper cooling of vacuum chamber and SR absorbers after wiggler is also required.

\section{REFERENCES}

[1] V.Korchuganov, M.Blokhov, M.Kovalchuk et al. "The status of the Kurchatov center of SR", Nuclear Instruments and Methods, A 543 (2005) pp. 14-18.

[2] A.Batrakov, I.Ilyin, G.Karpov et al. "Control and data acquisition systems for high field superconducting wigglers", Proceedings of $7^{\text {th }}$ International Conf on Synchrotron Radiation Instrumentation, Berlin, 2000.

[3] A.Batrakov, V.Jurba, S.Khrushchev et al. "A superconducting $3.5 \mathrm{~T}$ multipole wiggler for the ELETTRA storage ring", Proceedings of EPAC2002, Paris, pp. 2634 - 2636.

[4] S.Khrushchev, M.Kuzin, N.Mezentsev et al. "7 Tesla 17-pole superconducting wiggler for BESSY-II", Proceedings of XVIII International Workshop on Charged Particle Accelerators, Alushta, Ukraine, 2003 